\title{
XX. Nanotoxicity
}

Today nanoparticles and nanomaterials are used in many applications and commonly used in many areas, e.g., health care, electronics, chemistry, foodstuffs, automotive manufacture, energy production, cosmetics, fabrics, sensors, etc. Workers and consumers are often exposed to nanoparticles and nanomaterials. Assessing the risk on human health and the environment generated by these technologies is important but not simple because nanoparticles or nanomaterials can have properties different from those of macroscopic samples of the same materials. They are generally more reactive because they have a much larger surface of interaction.

In vitro and in vivo experiments are carried out to evaluate the toxicity of nanoparticles and nanomaterials. However, there are many controversial data in the literature and conclusions are often difficult to draw. In preparing this chapter, we have relied heavily on several review articles on this subject ${ }^{14}$. We refer the reader to these references to get a deeper insight into the state of the art in nanotoxicology.

\section{Hazard and risk}

Before we go any further it is worth recalling the difference between hazard and risk.

A hazard is a potential source of damage to a person, to equipment or to an organization. For example, when you are biking you can fall from your bicycle. A risk is the possibility that harm can result from a selected action or exposure to a particular hazard. The level of risk can often be quantified as a probability that harm will occur. Low risk means the probability of harm is relatively small. High risk means that the probability is large.

The level of risk depends both on the hazard and on your degree of exposure to the hazard. If you never use a bicycle, you have no chance to fall down. Loosely speaking, the level of risk is a quantity equal to the product of the degree of exposure to the hazard, times the seriousness of the hazard itself. For example, all things being equal, the risk of falling from a bicycle increases when biking more and more. Of course, without hazard, there is no risk.

Actually, zero risk doesn't exist. In any assessment of risk versus reward, the advantages and disadvantages of a given action should be evaluated 


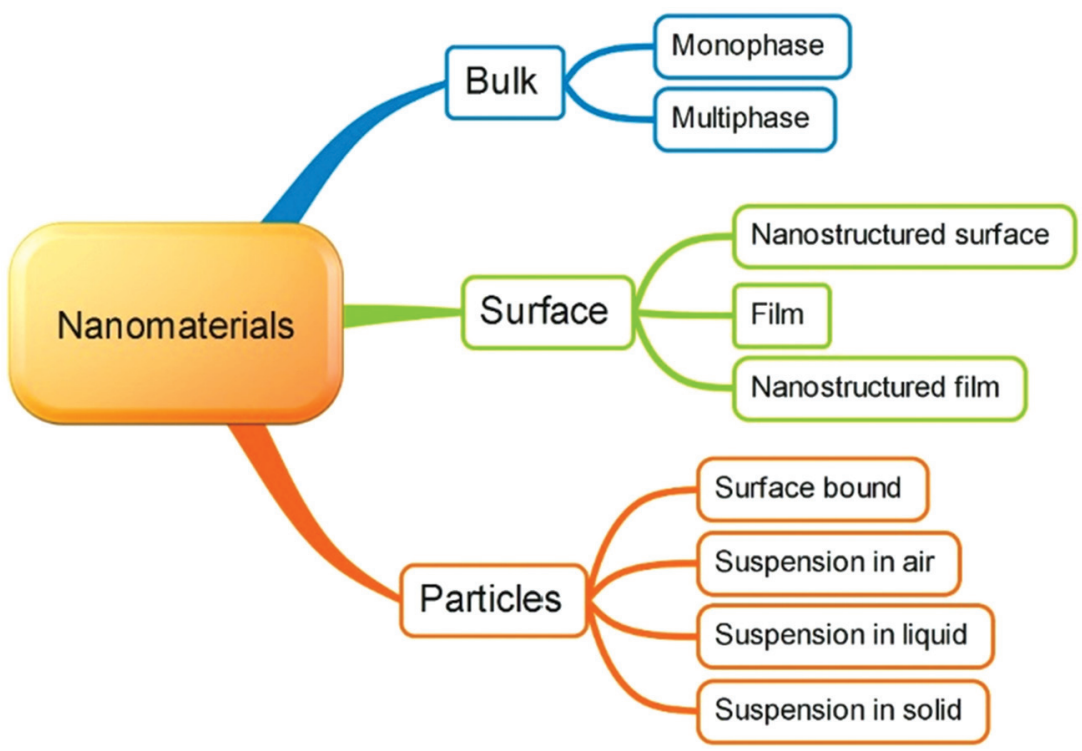

Figure 149. Classification of nanomaterials according to their location in an object.

and understood. This issue exists in most human activities and requires a careful analysis. Effort should be made to reduce risk as much as possible.

\section{Nanomaterials and nanoparticles}

The term nanomaterials encompasses materials composed of nanoparticles and those which are structured on the nanometer scale. A schematic means of classification of nanomaterials is summarized in figure 149. Nanomaterials can form the bulk of the material either as a single phase or as multiple phases (mixtures). Very often nanomaterials can be found on the surface of macroscopic objects providing them with specific properties. This can be a nanofilm deposited or built on a macroscopic surface, or a nanostructuration of the surface. For both bulk and surface nanomaterials, the nanoparticles or nanostructures are bound to the macroscopic object and give novel properties to the object, e.g., enabling photocatalysis to destroy harmful organic chemicals.

When the nanoparticles are embedded in a material which is part of a consumer product, the risk is usually very small for the consumer. Nanoparticles are trapped in the material and can be released only by wear or abrasion.

Nanoparticles can also be disbursed as suspensions in air, liquids or solids. 


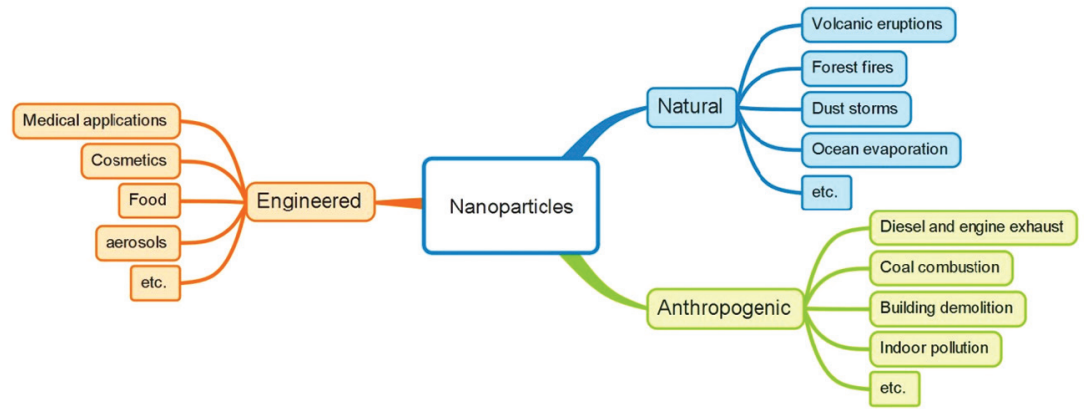

Figure 150. Nanoparticles can be of natural origin or man-made. For man-made nanoparticles one can distinguish between anthropogenic particles which are a byproduct of human activities and engineered nanoparticles which have been purposely manufactured for a specific application. Some domains of the different nanoparticles are indicated for illustration.

\section{Nanoparticle sources}

Nanoparticles can have different origins:

- They can come from natural processes such as volcanic eruptions or forest fires

- They can be emitted during human activities such as combustion of coal or diesel fuel. We shall say that they are from anthropogenic origin: These are man-made but not created on purpose.

- Nanoparticles can also be produced on purpose and used because of their specific properties. This is the case for example of functionalized nanoparticles used in therapeutics, or nanoparticles such as $\mathrm{TiO}_{2}$ used in cosmetics, toothpaste or sun creams. These nanoparticles are of anthropogenic nature. We shall put them in the special category of engineered nanoparticles. Figure 150 shows our classification scheme with a few representative examples of the domains in which they are encountered.

\section{Nanoparticle exposure}

Exposure to certain nanoparticles may have an adverse effect on human health Airborne nanoparticles are certainly those which present the largest potential hazard to human health. Free nanoparticles are usually engineered in such a way that they do not aggregate with each other. If nanoparticles penetrate into some part of the body they can induce health problems. 


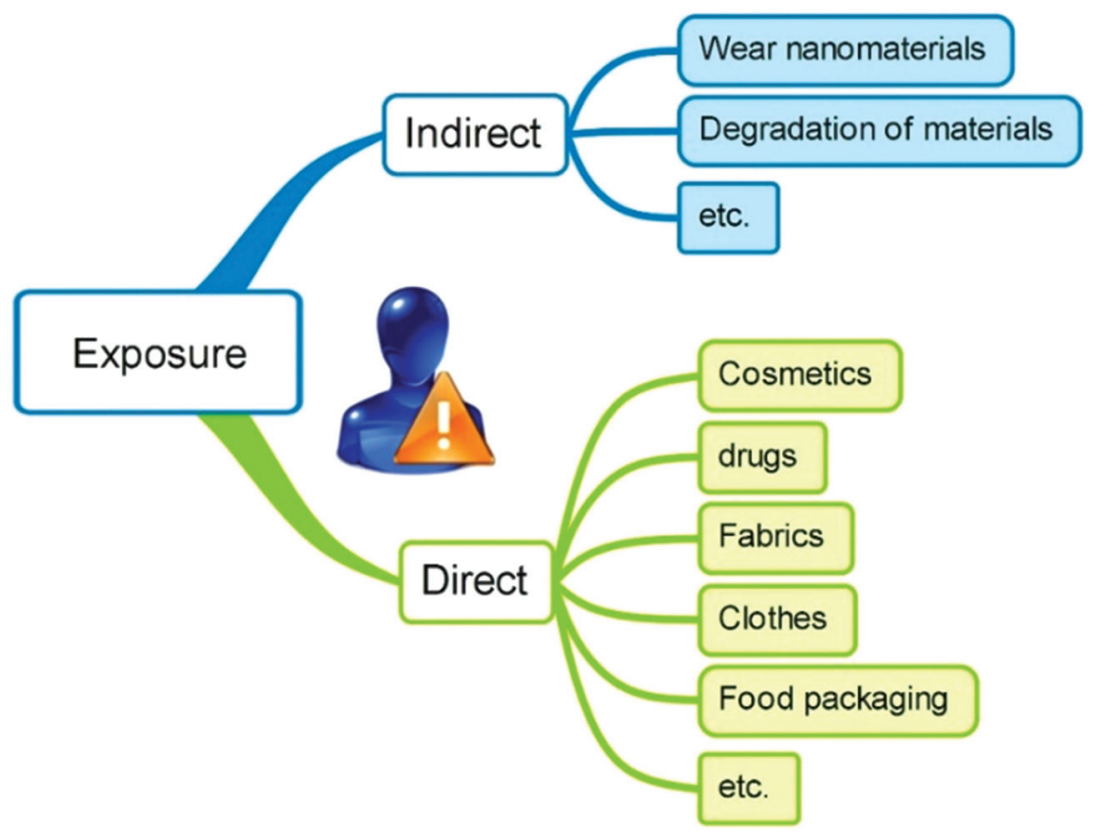

Figure 151. Direct and indirect exposure to nanoparticles.

The exposure to nanoparticles may be direct or indirect (figure 151). There is a direct exposure when the nanoparticles are intended for contact with the consumer. This is true of cosmetics, drugs, fabrics, clothes, food packaging, etc. Indirect refers to non-intended exposures which might occur during the use of an object containing nanomaterials. Such exposures might come from surface coatings of the objects, or from their degradation at the end of their life.

Nanoparticles can enter the human body by several routes. These are displayed in figure 152: inhalation, penetration through the skin or wounds, by ingestion or through the parenteral route (intravenous, intra-arterial, intra-osseous, intramuscular, intrathecal). Since the skin, lungs and the gastro-intestinal tract are in contact with the environment, they are potentially the easiest entry points for nanoparticles.

\section{Toxicity of nanoparticles}

Workers can be faced with high concentrations of nanoparticles during the manufacturing process. Inhalation is a favored route of contamination, but 


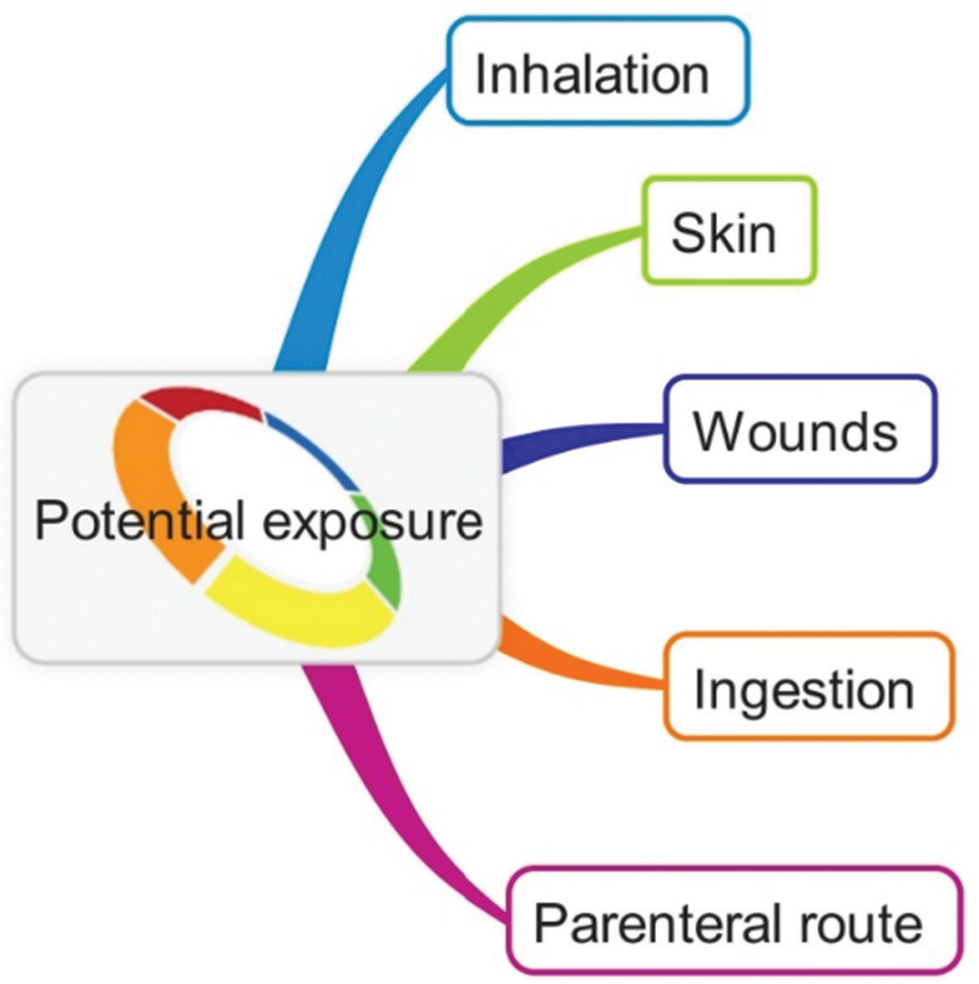

Figure 152. Routes that can be taken by nanoparticles to enter the body.

skin and wound penetration are also efficient pathways for the nanoparticles to penetrate the human body. The workers should be protected against any direct interaction with the nanoparticles. At the manufacturing level it is relatively easy to design an industrial installation to prevent worker contact with nanoparticles using appropriate protective clothing, filtration and ventilation techniques. Product recovery, cleaning, equipment maintenance, packaging processes and storage are also steps leading to potential worker exposure to nanoparticles. At the industrial level and with good standards, this problem can be solved. It is, however, very difficult to control the full life-cycle (production, transport, storage, usage and disposal) of an object containing nanomaterials. There is a risk for consumers that must be evaluated and minimized.

Evaluation of the toxicity of nanoparticles is a complex and difficult problem. Some health disorders may only appear a long time after exposure. Most toxicology studies are carried out in vitro or in vivo on animals or cells and high concentrations of nanoparticles are used. These concentrations do 
not necessarily correspond to those normally encountered. Establishing permissible dose levels is an important imperative that requires deeper studies.

To illustrate the point - there have been toxicity studies of the effect on human cells of iron based magnetic nanoparticles as well as of silver and zinc oxide nanoparticles. A summary of these studies has been published by Auj-E Taqaddas ${ }^{15}$. Magnetic iron nanoparticles have several potential applications in medicine such as contrast agents in diagnostic, targeted drug delivery or hyperthermia. They are considered safe because of their biocompatibility and the fact that an excess of these nanoparticles is eliminated by the liver and the spleen. However, it has been shown in tests on specific breast cancer cells that these nanoparticles can damage the membrane of the cell. The extent of the damage depends on the concentration of nanoparticles, on their sizes and on the contact time. The nanoparticles induce an intracellular stress on the cells that damages DNA and can lead to apoptosis (death) of the cell. It is possible to reduce the toxicity of the nanoparticles by surface coating them, for example with gold or by a proper functionalization of the surface.

Silver nanoparticles are widely used in particular because of their anti-microbial and biocidal activities but they also have interesting properties such as high thermal and electrical conductivities. They are increasingly used, especially in medicine and in fabrics. Because of their toxic properties to organisms, it must be verified that the use of silver nanoparticles is safe for health and the environment.

In the health area, although they have been used for many years, it seems that silver nanoparticles are safe as long as they are used at low dose. The use of silver nanoparticles in fabrics has been approved in many countries. A problem is that fabrics can release some of these nanoparticles into the environment, during washing for example.

The potential diseases that can be associated to exposures to nanoparticle are summarized in figure 153 (Critina Buzea et al). This chart has been deduced from the results of in vivo and in vitro studies. It summarizes possible health problems associated with inhalation, ingestion and contact with nanoparticles. This figure corresponds to the worst situation that can be imagined.

The toxicity of nanoparticles depends on several factors such as their size, shape, composition, functionalization, structure, crystallinity, etc. It also depends on the biological properties of the organism, the pathway followed by the nanoparticles and their nature.

- Inhaled nanoparticles can lead to asthma, bronchitis, emphysema, lung cancer, and neurodegenerative diseases (Parkinson's, Alzheimer...). 


\section{DISEASES ASSOCIATED TO NANOPARTICLE EXPOSURE}

C. Buzea, I. Pacheco, \& K. Robbie, Nanomaterials and nanoparticles: Sources and toxicity, Biointerphases 2 (2007) MRI7-MR7I

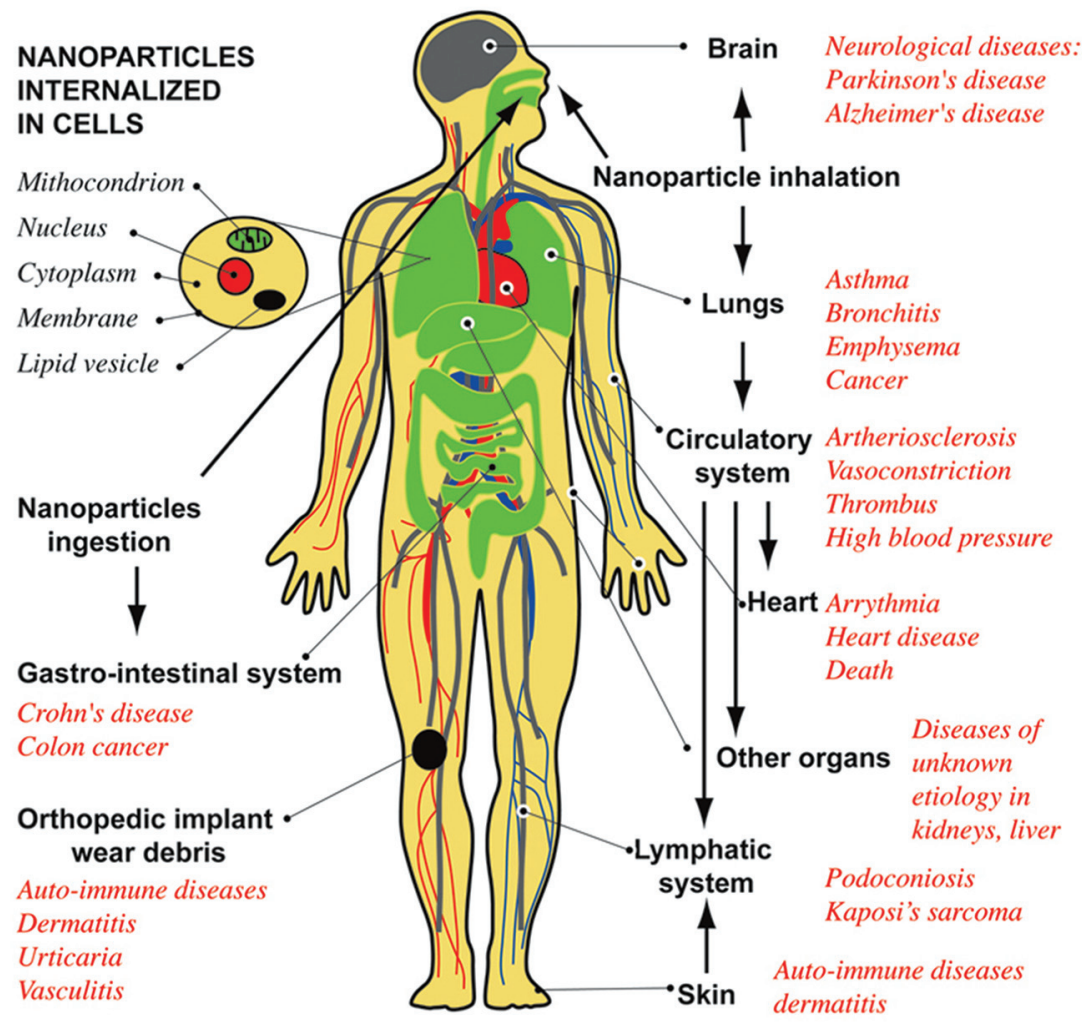

Figure 153. Pathways of exposure to nanoparticles and associated diseases as suggested by epidemiological, in vivo and in vitro studies. Image from Wikimedia Commons (http://commons. wikimedia.org), Cristina Buzea.

- In the gastrointestinal tract, nanoparticle ingestion can lead to Crohn's disease and colon cancer.

- Nanoparticles can also enter into the circulatory system with the possibility of inducing arteriosclerosis, blood clots, arrhythmia, heart diseases and possibly cardiac death.

Translocation to other organs (liver, spleen, brain...) may induce other diseases. Furthermore, some nanoparticles are suspected of inducing autoimmune diseases.

This description of adverse health effects can be scary but relates to only a small number of the natural nanoparticles found in the environment or 
those which are man-made. Many nanoparticles are non-toxic and the consequences on health care shown in figure 153 do not apply. It is also possible, starting from a nanoparticle that is initially toxic, to modify it to make it non-toxic. Furthermore, nanoparticles can aggregate locally, producing larger sized particles that are less dangerous for health.

In the following three sections we discuss some of the health issues associated with the three different classes of nanoparticles identified in Figure 150.

\section{Natural nanoparticles}

There are naturally occurring nanoparticles (1-10o $\mathrm{nm}$ in size). These nanoparticles, not produced by human activities, are mostly found in hydrous environments. Produced by forest fires, volcanic eruptions, dust storms or similar events. These nanoparticles may be found in streams, can be carried by the wind and dispersed into the environment. They are present in large numbers in each breath we take and in our drinking water.

Less violent natural processes can also produce nanoparticles ( $\mathrm{ZnS}$, $\mathrm{Fe}_{3} \mathrm{O}_{4}$, metal sulfides...). For example, zinc sulfide (ZnS) nanoparticles are formed by microbial action. More generally, other natural processes, for instance, drainage in acid mine sites are able to produce mineral nanoparticles. Generally, most natural nanoparticles have a low toxicity.

At the global level, most atmospheric aerosols are produced by wind erosion. Dust storms can travel over long distances. For example, the particles of dust storms occurring in the Gobi Desert can reach North America. The size of the particles transported is generally between $100 \mathrm{~nm}$ and several microns. Particles between $100 \mathrm{~nm}$ and $200 \mathrm{~nm}$ can be found at concentration around 1,500 particles $/ \mathrm{cm}^{3}$. These airborne dust particles can induce health problems with people subject to asthma and emphysema. The lung tissue can also be affected thanks to iron and other metal particles present in the dust.

Ashes and gases containing particles with sizes ranging from the nanoscale to the microscale emitted in volcanic eruptions, cause respiratory problems, eye and skin irritation. Traveling to very high altitudes, they spread over the world and can affect areas of the planet for years following an eruption. Populations living barefoot in areas with a volcanic soil are affected by serious diseases (podoconiosis, lymphedema, Kaposi's sarcoma, etc.) probably coming from the absorption through the soles of nano and microparticles coming from the soil. 
A large quantity of aerosols is emitted from the seas and oceans. Sea salt particles with dimensions from $100 \mathrm{~nm}$ to several microns can be found in these aerosols. Unlike other kinds of aerosols, they have a positive effect on health and can relieve some respiratory problems.

Nano-organisms such as bacteria, viruses and other organisms can have an impact on human health. They are probably the cause of some neurodegenerative diseases. Nanobacteria could be involved in diseases linked to calcification such as artery plaque, aortic aneurysm, renal stone formation, prostatitis, ovarian and breast tumors, etc.

Gas molecules and viruses can have a size in the 1-100 nm range. For example, the HIV (human immunodeficiency virus) is about $100 \mathrm{~nm}$ diameter. Molecules and viruses can be present in the environment, especially in the air, but also in water. Viruses interact with biological systems and exploit cellular processes to replicate themselves. Some viruses or nanobacteria have seriously adverse health effects on people. Some chronic diseases that were thought to come from lifestyle or genetic factors are actually due to viruses. For example, cervical cancer is due to the Papilloma virus and the nasopharyngeal cancer comes from the Epstein-Barr virus.

The effect on health of inert nanoparticles present in the environment should not underestimated compared to viruses despite the fact that they do not have the ability to take control of a cell and replicate themselves. Their very small sizes give them the ability to enter the organism and translocate within it. In the worst case, within the organism, these particles can cross physiological barriers (air-blood barrier in the lungs, blood-brain barrier or materno-foetal barrier) and travel through the circulatory system (blood circulatory, lymphatic system). Skin, lungs and the gastro-intestinal tract are in contact with the environment. They are potentially good entry points for nanoparticles.

Some nanoparticles can penetrate inside cells and induce damage. They can modify processes of the cell. Some diseases may result from the perturbation of the cell operation. For example, cancer, which is an uncontrolled proliferation of cells, can be induced as can neurodegenerative diseases which are due to a premature death of cells. Autoimmune diseases can also be triggered by modifications of the cell functions.

The outer membrane of a cell, which is a phospholipid bilayer, can be affected directly by a nanoparticle, leading to a disruption of the cell membrane such as a nanohole punched into the membrane. The membrane can also be affected indirectly, for example by oxidative or surfactant damage, or damaged by toxic ions. The interaction of a nanoparticle with a membrane 
depends very much on the properties of its surface. Inside the cell, nanoparticles can perturb the cell's constituents. In the nucleus, the DNA can be damaged. The lysosome and mitochondria can also be damaged and there can be a lipid peroxidation in the vesicles.

\section{Anthropogenic nanoparticles}

There are also nanoparticles present in the environment which are not produced on purpose, but produced in normal human activities. These are called anthropogenic nanoparticles. They result from industrial activities such as emission from the exhaust pipes of automobiles and trucks, the production of electricity using coal plants or in the smoke from cigarettes. In large cities it is common to have a few hundred million nanoparticles per liter of air. In discussing this category, we exclude engineered nanoparticles which have been manufactured on purpose for a specific application. These are treated in the following section.

Vehicles, especially those using diesel engines, are the main source of particles (micro and nano) in urban areas. Most of the particles emitted from the exhaust pipes have sizes in the range of $20-60 \mathrm{~nm}$ for diesel engines and 20-130 $\mathrm{nm}$ for gasoline engines. These particles are almost spherical. However, in the case of diesel engines, carbon nanotubes and fibers have been found as byproducts of the combustion. Since their shapes are very similar to those of asbestos fibers, there is a non-negligible probability that they could be carcinogens. Air pollution from transportation exhaust is a serious health problem and there is a correlation between the concentration of nanoparticles near the roads or highways and an increased rate of cardiopulmonary mortality for those people living very close to them. Nanoparticles increase the risk of cardiovascular events.

Burning coal in power plants produces carbon and sulfide particulates and, surprisingly, uranium dioxide $\left(\mathrm{UO}_{2}\right)$ nanoparticles. Actually, if no efficient filter is used in the exhaust chimney, the radioactivity emitted by burning coal is larger (by a factor which can be of the order of one hundred, depending upon the origin of the coal used) than that emitted from an operating nuclear plant. A good filter for the smoke emitted by a coal plant can become a notably radioactive object.

Tobacco smoke is a complex mixture containing more than 100,000 compounds. Many of these are health hazards. It also contains particles with sizes ranging from $10 \mathrm{~nm}$ to $700 \mathrm{~nm}$, the average being around $150 \mathrm{~nm}$. 
Tobacco smoke greatly increases the risks of developing chronic respiratory illnesses, lung, pancreatic or nasal cancers, and cardiovascular diseases.

Particles are also emitted in the demolition of large buildings. Some of them are toxic, especially when old buildings are demolished because they can contain asbestos fibers or lead. The dust cloud generated during demolition can travel tens of kilometers from the demolition site. Following the attack on the World Trade Center, on September 2001, many first responders suffered respiratory problems. More than 5,000 cancers are attributed to that attack.

Indoor pollution is notably greater than outdoor pollution. Several inside activities create nanoparticles: cooking, smoking, cleaning, etc. Nanoparticles are also produced by living organisms: skin particles, dust mite droppings, etc. Since many people now live more indoors than outdoors, indoor pollution is a real issue.

In some countries in Asia or Africa, indoor smoke emitted from the use of coal, biomass, wood and other fuels is responsible for the deaths of about 1.6 million people per year, half of them children under five years old.

\section{Engineered nanoparticles}

Many kinds of engineered nanoparticles are now produced, some of them in large quantities. Tens of thousands of tons of silica, alumina, black carbon, titanium dioxide nanoparticles and nanoclays are produced every year. Hundreds of tons of nanoparticles of metal, ceramic-metal, rare earth and carbon nanotubes are also produced yearly. Other nanoparticles are produced in smaller quantities. In contrast to the anthropogenic nanoparticles that we discussed previously, engineered nanoparticles are designed to satisfy specific functions. For that they must remain stable, at least until used. Most of these nanoparticles, around $45 \%$, are used in industrial materials while about $3 \%$ are reserved for medical applications. The increasing use of nanoparticles in medical applications demands that they be made as safe as possible. The design of the surfaces of nanoparticles used in drug delivery to increase uptake into cells is an important factor in assuring safety.

Cosmetics is an area where engineered nanoparticles are extensively used. Actually the first use of nanoparticles in cosmetics can be traced back to the black soot and mineral powders used in ancient Egypt. Today nanoparticles with specific optical properties or functions such as antioxidant, peptide or nutrient delivery are employed in creams, lipsticks, toothpastes, 
shampoos, hair conditioners, etc. The advantage of nanoparticles is that they can easily penetrate deeply into the protective layers of the skin. Functionalized fullerenes can now be found in creams because of their supposed free-radical scavenging properties. Alumina nanopowder is also used in cosmetics because it is supposed to reduce small wrinkles.

Very stable titanium dioxide $\left(\mathrm{TiO}_{2}\right)$ nano or microparticles are also widely used in many consumer products: sunscreens, cosmetic creams, food colorants, toothpastes, pharmaceuticals, etc. $\mathrm{TiO}_{2}$ is widely used as a white pigment because of its brightness and high refractive index. About four million tons of this pigment is produced every year worldwide. That represents about $70 \%$ of the total amount of pigments produced in the world. Surprisingly, it can be used to whiten skim milk.

$\mathrm{TiO}_{2}$ nanoparticles are anticorrosive and have photocatalytic properties. This photocatalytic effect is important in several applications where selfcleaning and antifogging properties are needed: self-cleaning windows, tiles, textiles, and antifogging car-mirrors.

$\mathrm{TiO}_{2}$ particles with diameters larger than $100 \mathrm{~nm}$ are considered to be biologically safe for humans and animals. It seems almost sure that $\mathrm{TiO}_{2}$ nanoparticles cannot penetrate the intact skin into the human body. On the other hand, intravenous injection of large quantities of $\mathrm{TiO}_{2}$ nanoparticles can induce pathological lesions of the liver, spleen, kidneys and brain.

Silver nanoparticles have the ability to kill bacteria and microorganisms. Because of this property, they are widely used to impregnate consumer products such as socks, underwear, bedding, sportswear, toys, wound dressing, etc. During use and laundering, silver nanoparticles can be released into the environment, especially in wastewater. Silver nanoparticles are also used in medicine. As for many other nanoparticles, they can cause oxidative stress of cells. The toxicity of silver nanoparticles has been investigated in special cases but there are still large uncertainties to be resolved.

Fullerenes and carbon nanotubes, both single wall and multiwall, are now used in several applications. Published work in the scientific literature is ambiguous because some indicate that carbon nanotubes are highly toxic while others indicate that they not. In vivo studies on animals have been carried out. The toxicity depends upon the length of the nanotube and on its functionalization. Often the toxicity results obtained cannot be easily related to carbon nanotube usage because the usage conditions are different and the carbon nanotubes are different. Further studies are required. 


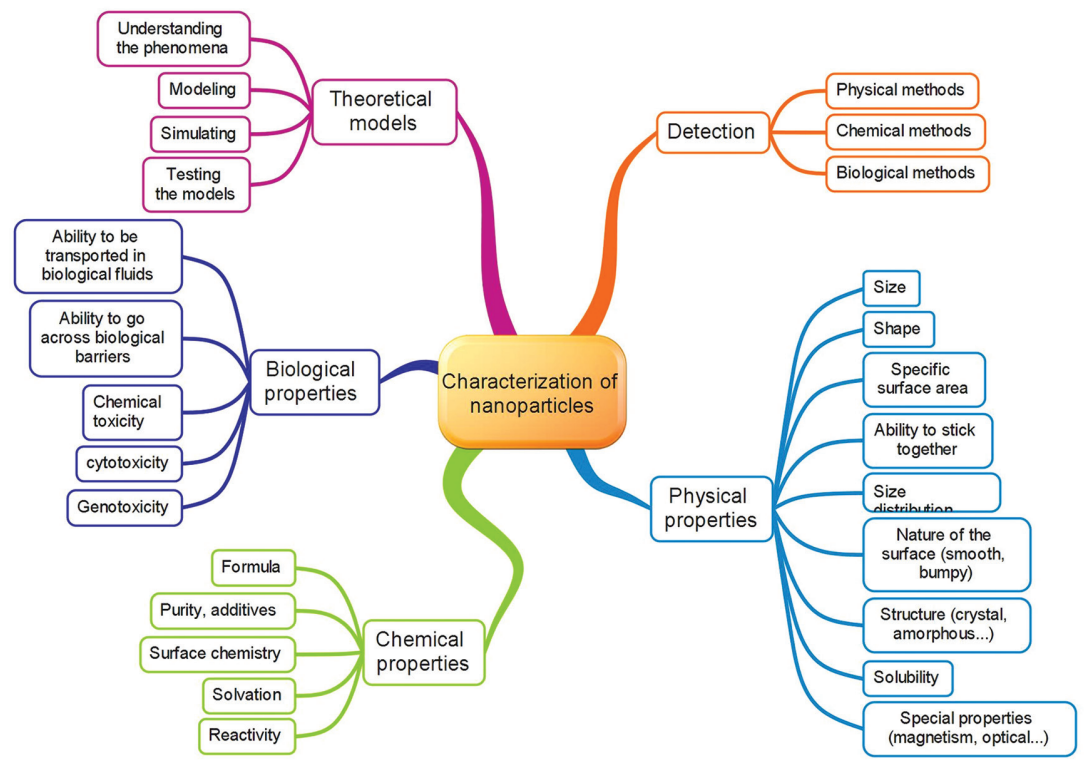

Figure 154. Main areas of investigation to the determination of the toxicities of nanomaterials and nanoparticles.

\section{Summary}

Humans have been exposed to nanoparticles throughout human history. Not all the nanoparticles with which people are in contact lead to serious adverse health effects. It even turns out that some nanoparticles are good for health. This the case for those generated from evaporation of the seas.

The industrial revolution, with its increased combustion of fossil fuels, greatly boosted concentrations of man-made nanoparticles in large cities as well as in many other parts of the world. Many of these have adverse health effects.

The wide-spread use of engineered nanomaterials and nanoparticles adds new risks that must be carefully investigated and balanced with the advantages obtained by their use. While the manufacturing of nanomaterials can be accomplished in working conditions that are safe for the workers and the environment, little is known about long-term exposure of consumers to low concentrations of engineered nanoparticles because it is a rather novel domain. The problem is complex. The interaction mechanisms between engineered nanoparticles and living systems are far from being completely understood. Many parameters are involved and not only the mass or concentration of the nanoparticles. Several aspects have to be 
investigated to get a better understanding of nanotoxicity. Certainly long term epidemiological studies are needed.

The toxicity limits of bulk materials are usually specified in mass units. This parameter is sufficient to characterize the hazard of a material at macroscopic scale. For example, we know that absorbing 100-200 mg of potassium cyanide will kill a normal person. However, for nanoparticles, the mass is not the only relevant parameter. The adverse health effects of nanoparticles depend on many other parameters such as the surface to volume ratio, the shape, the composition, etc. Generally, the smaller the nanoparticle the more toxic it is. However, each situation can be different and nanotoxicology studies have to be carried out or modeled using relevant experimental results. Last but not least, the translocation of nanoparticles inside the body and the interaction with cells are important subjects that need to be fully understood. Since nanomaterials and nanoparticles have different properties from bulk materials, the results of toxicity studies done on bulk materials at the macroscopic scale are not sufficient to describe their toxicities The main areas of investigation relevant to the appropriate determination of the toxicities of nanomaterials and nanoparticles are presented in figure 154 . 
